\section{Evaluation of retinopathy of prematurity screening in reverse Kangaroo Mother Care: a pilot study}

TR Padhi ${ }^{1}$, D Sareen ${ }^{1}$, L Pradhan ${ }^{2}$, S Jalali ${ }^{3}$, S Sutar ${ }^{1}$, T Das $^{3}$, RR Modi ${ }^{1}$ and UC Behera ${ }^{1}$

Eye (2015) 29, 505-508; doi:10.1038/eye.2014.340; published online 23 January 2015 prematurity (ROP) screening practice in reverse Kangaroo Mother Care (R-KMC) with respect to stress and pain to the infant.

Methods In a pilot study we evaluated ROP screening practice in R-KMC in 20 babies at risk of ROP. The R-KMC differed from the conventional KMC with respect to the baby position where the baby lay supine on mother's chest. With the mother lying supine and the baby in R-KMC position, screening examinations were done with indirect ophthalmoscope. The outcome measures included stress (quantified by pulse, respiration, and oxygen saturation) and pain to the baby by observing facial expression (eye squeezing, crying, and brow bulge). The heart rate, respiratory rate, and SpO2 (\%) were compared before and immediately after the procedure using paired $t$-test. Result Mean ( \pm SD) gestational age and birth weight were $30.8 \pm 2.3$ weeks and $1362.5 \pm 253.9 \mathrm{~g}$, respectively. During examination in R- KMC position 8 babies $(40 \%)$ were completely relaxed (no eye squeezing and crying), 10 (50\%) were partially relaxed (no brow bulge) and 2 babies (10\%) were not relaxed. A change in heart and respiration rate both by 10 per minute was recorded in $12(60 \%)$ and $10(50 \%)$ babies, respectively. Five babies (25\%) had reduction in blood oxygen concentration below $92 \%$. The majority of the mothers (19 of 20) were relaxed. Conclusion ROP screening in R-KMC can be a baby friendly screening practice with respect to stress and pain to the infant and needs further evaluation in a larger cohort.

\section{Introduction}

Timely screening and prompt treatment have been the mainstay of management of retinopathy of prematurity (ROP). Examination of at-risk-babies with indirect ophthalmoscope is the current gold standard. Several reports suggest possibility of adverse physiological changes (increased heart rate and decreased oxygen saturation levels) ${ }^{1-6}$ or increased stress and pain to the infant (measured from facial expression of the baby) during eye examination. This is one of the reasons for the International Evidence-Based Group for Neonatal Pain to include eye examinations for ROP in the list of painful procedures performed in the NICU. ${ }^{7}$ Suggestions to minimize neonatal discomfort from ROP examinations include avoiding use of eye speculum, ${ }^{8}$ gentle and quick examination in a dark and calm environment, ${ }^{9}$ pre-examination topical anaesthesia, ${ }^{10}$ pacifiers like oral sucrose, ${ }^{2}$ nonnutritive sucking, and swaddling and holding or nesting the baby during fundus evaluation.

In recent years Kangaroo Mother Care (KMC) has evolved into a better practice scenario for care of premature babies. This concept originates from care of the baby kangaroos who are born very immature, as are human 'premature' babies. Typically in KMC, the baby wears only a diaper and is tied in a head-up position to the mother's bare chest with a strip of cloth (Figure 1). It has numerous advantages for the infant such as better control of temperature, longer sleep, decreased cry, stable cardiac and respiratory activities, less apneic episodes, less nosocomial infection, and later better weight gain. The maternal contact
${ }^{1}$ Retina-Vitreous Service, LV Prasad Eye Institute, Bhubaneswar, India

${ }^{2}$ Infosys Pediatric Center, Capital Hospital, Bhubaneswar, India

${ }^{3}$ Srimati Kanuri Santhamma Center for Vitreoretinal Diseases, LV Prasad Eye Institute, Hyderabad, India

Correspondence: TR Padhi, Retina-Vitreous Service, LV Prasad Eye Institute, Patia, Bhubaneswar, Odisha 751 024, India Tel: +91 9438361078; Fax: +916743987130 E-mail:drtapasranjan@ yahoo.co.in

Received: 26 September 2014

Accepted in revised form: 12 December 2014

Published online:

23 January 2015

Institution at which the study was conducted: Infosys Pediatric Center, Capital hospital,

Bhubaneswar, India. 

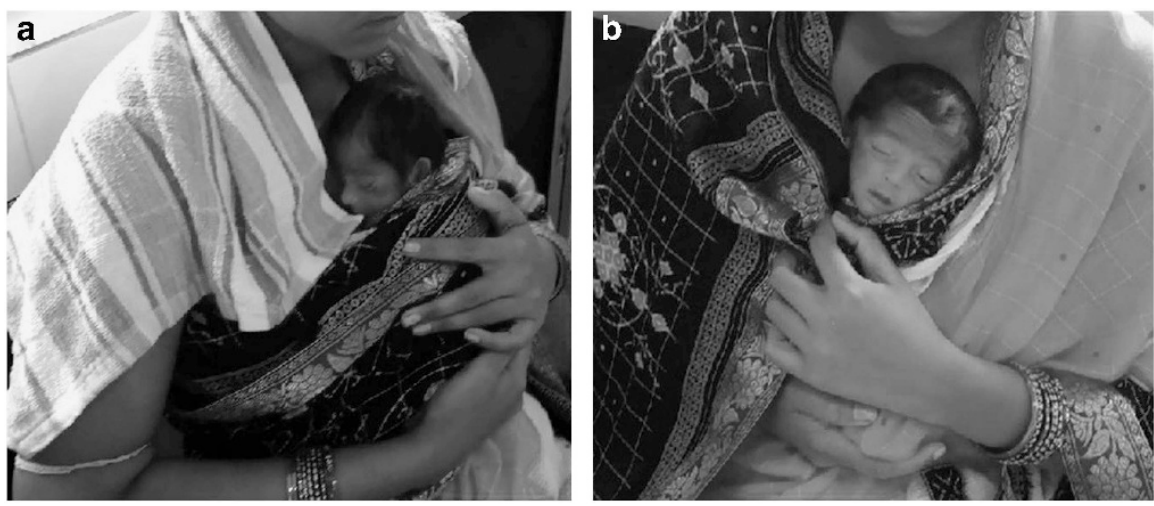

Figure 1 (a) Baby position in conventional Kangaroo Mother Care where the baby stripped of its clothing except diaper rests on the mother's bare chest in a frog position. A piece of cloth wraps and holds the baby against the mother assisted by the support from the arms of the mother. The head lies in a lateral position making the fundus examination inconvenient. (b) Reverse Kangaroo Mother Care position where the baby is rotated $180^{\circ}$, other formalities remaining the same. Here the baby's eyes look straight ahead making the fundus examination easy and convenient.

causes a calming effect along with reduced physiologic and behavioural pain responses. ${ }^{11}$ Currently KMC is used as a pain reliever for many procedures like intravenous cannulation, blood withdrawal, and heel prick. ${ }^{12}$ While we exploited these advantages in our ROP care, we made a slight modification to the traditional KMC posture by reversing the position of the baby's face in relation to mother's chest. We called this reverse KMC (R-KMC).

\section{Materials and methods}

This prospective study was conducted at the neonatal unit of Capital hospital, Bhubaneswar, India over a period of 6 months. The study was approved by the ethics committee, LV Prasad Eye Institute, Bhubaneswar and adhered to the tenets of the declaration of Helsinki. Twenty babies at risk of ROP were screened for ROP in R-KMC after due consent and parent counseling. The babies were fed $30 \mathrm{~min}$ before the procedure and the eyes were dilated with ROP dilating medications (2.5\% phenylephrine and $0.5 \%$ tropicamide). The mother was counseled and informed in detail about the technique of KMC and R-KMC. In traditional KMC (Figure 1a), the baby's head lies in a lateral position and hence fundus examination becomes very difficult. In R-KMC the baby was placed supine over the mother's chest (Figure 1b) that maintained a direct contact with baby's back. A KMC cloth/strap was used to wrap the baby and the mother. Mother was advised to keep her hands one over the chest of the baby and restrain two arms while the other hand restrained both the legs. This restrained the baby adequately with the eyes facing straight ahead towards the examiner. After this formal training, the mother was asked to lie supine on the examination couch and the baby was placed in a R-KMC position as already described. The baseline blood oxygen concentration, respiration and pulse rates were recorded. Topical anaesthetic (Proparacaine hydrochloride 0.5\%) was applied to both eyes. The eye speculum was inserted gently by the ophthalmologist after $30 \mathrm{~s}$ while the assistant restrained the head by keeping her palms gently on either side of the head of the baby (Figure 2a). Both eyes were then examined one after another by indirect ophthalmoscope and wire vectis (for scleral indentation) by going around the bed on either side (Figure $2 b$ ). The pulse, respiration, and blood oxygen saturation were recorded again immediately after conclusion of the procedure. We ensured that the same individual was involved in all vital steps of examination, be it measurement of vital parameters by a paediatric trained nurse or be it the eye examination by the paediatric retina specialist. We enrolled only new patients (as opposed to review babies who need shorter time for examination) and this maintained uniformity. Statistical analysis was done by using the InStat statistical software version Win $3.0 \times$ (GraphPad Software Inc., La Jolla, CA, USA). The heart rate, respiratory rate, and $\mathrm{SpO} 2(\%)$ were compared before and immediately after the procedure using paired $t$-test.

\section{Results}

The mean $( \pm S \mathrm{SD})$ gestational age (GA) and birth weight (BW) of the 20 screened babies were $30.8 \pm 2.3$ weeks and $1362.5 \pm 253.9 \mathrm{~g}$, respectively. The mean $( \pm \mathrm{SD})$ post menstrual age (PMA) at examination was $33.9 \pm 2.9$ weeks. All stress and pain parameters could be recorded in each baby and each baby completed the screening procedure. The two-tailed $P$-values for change in heart rate and $\mathrm{SpO} 2(\%)$ were 0.9108 and 0.3965 , respectively, while that for respiratory rate was 0.0037 . The changes in heart rate and $\mathrm{SpO} 2(\%)$ were not statistically significant. 

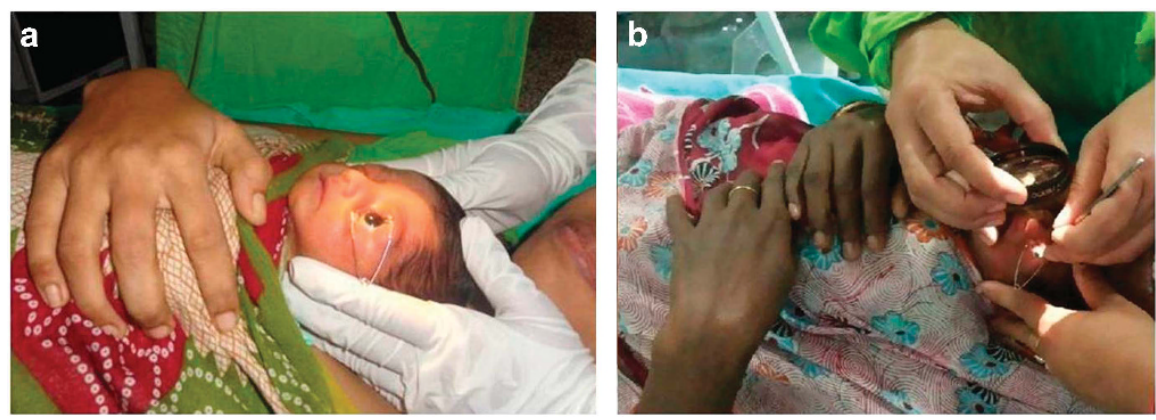

Figure 2 (a) Position of the mother and baby in reverse Kangaroo Mother Care position just before examination. (b) Retinopathy of prematurity screening with indirect ophthalmoscope in reverse Kangaroo Mother Care position where the mother and the baby lie supine on the examination couch. The infant's eyes, with pupils pharmacologically dilated, are held open by an eyelid speculum. The mother restrains the baby with her upper and lower arms while an assistant holds the head steady for the ophthalmologist to view the retina with an indirect ophthalmoscope and $20 \mathrm{D}$ lens.

But the change in respiratory rate was statistically significant.

Besides the above statistical tests, for a better clinical relevance, we also analysed the data in another way. We arbitrarily fixed the change in heart and respiration rate by $10 / \mathrm{min}$ (either rise or fall) and fall in blood oxygen saturation below $92 \%$ as significant and indicative of stress to the infant. We calculated the number and percentage of infants having these significant changes as well as their behavioural changes, which is summarized in Table 1. Moderate-to-severe discomfort evidenced by eye squeezing, crying, and brow bulge during the procedure was noted only in two babies while only one mother was anxious during the examination. Majority of those babies, who cried, stopped crying just after or within $10 \mathrm{~s}$ of the conclusion of the examination.

\section{Discussion}

The results indicated that when examined in R-KMC, most babies were comfortable or had transient mild-to-moderate stress or pain. The stabilization was early even in those who were distressed during examination. Though the study did not aim at the efficacy of the procedure in detecting retinopathy, examination in reverse KMC was as effective as our conventional technique.

We recognize few important limitations in this study. (1) This series is small and hence warrants a larger cohort. (2) This study did not compare the stress profile with respect to the conventional method and warrants an adequately powered randomized controlled trial to address the same. Neonates are more sensitive to pain than adults. ${ }^{13,14}$ This pain sensitivity is further accentuated in preterm neonates and may not be clinically evident, ${ }^{14-16}$ as the critically ill and preterm neonates do not mount vigorous behavioural responses to pain. The pain modulation systems that operate in older children and adults do not appear to be fully
Table 1 Summary of the stress and pain parameters of the babies examined in reverse Kangaroo Mother Care

\begin{tabular}{lc}
\hline Change in stress parameters & No. of babies \\
\hline $\mathrm{HR}>10$ per minute & $12(60 \%)$ \\
$\mathrm{RR}>10$ per minute & $10(50 \%)$ \\
Both HR and RR change $>10$ per minute & $05(25 \%)$ \\
& \\
Behavioural changes & $8(40 \%)$ \\
No eye squeezing, no crying & $4(20 \%)$ \\
Only eye squeezing & $6(30 \%)$ \\
Eye squeezing and crying & $2(10 \%)$ \\
Brow bulging and crying &
\end{tabular}

Abbreviations: HR, heart rate; RR, respiration rate.

functional in newborns. Therefore, a stress score that addresses correction factors for GA or PMA like premature infant pain profile (PIPP) tool ${ }^{17}$ would have been more informative and is the third limitation.

On the basis of our pilot study, we suggest that R-KMC screening could be considered for further evaluation in ROP screening practices.

\section{Summary}

What was known before

- Retinopathy of prematurity (ROP) screening examinations are associated with stress and pain to the infants.

- Many behavioural and pharmacological measures reduce this stress and pain to certain extent but our search for a baby-friendly screening practice continues.

- Kangaroo Mother Care (KMC) has evolved as a better practice pattern for neonatal care and can be used to relieve procedural pain in infants.

What this study adds

- Reverse KMC (baby is rotated 180 degree and lies supine on mother's chest) is a novel concept that can facilitate eye examination in neonates.

- ROP screening in reverse KMC position is one step towards making the examination baby friendly and relaxing for the mother. 


\section{Conflict of interest}

The authors declare no conflict of interest.

\section{References}

1 Boyle EM, Freer Y, Khan-Orakzai Z, Watkinson M, Wright E, Ainsworth JR et al. Sucrose and non-nutritive sucking for the relief of pain in screening for retinopathy of prematurity: a randomized controlled trial. Arch Dis Child Fetal Neonatal Ed 2006; 91: F166-F168.

2 Gal P, Kissling GE, Young WO, Dunaway KK, Marsh VA, Jones SM et al. Efficacy of sucrose to reduce pain in premature infants during eye examinations for retinopathy of prematurity. Ann Pharmacother 2005; 39: 1029-1033.

3 Kirchner L, Jeitler V, Pollak A, Müllner-Eidenböck A, Weinzettel R, Kraschl R et al. Must screening examinations for retinopathy of prematurity necessarily be painful? Retina 2009; 29: 586-591

4 Kleberg A, Warren I, Norman E, Morelius E, Berq AC, Mat-Ali E et al. Lower stress responses after Newborn Individualized Developmental Care and Assessment Program care during eye screening examinations for retinopathy of prematurity: a randomized study. Pediatrics 2008; 121: e1267-e1278.

5 Rush R, Rush S, Ighani F, Anderson B, Irwin M, Naqvi M. The effects of comfort care on the pain response in preterm infants undergoing screening for retinopathy of prematurity. Retina 2005; 25: 59-62.

6 Samra HA, McGrath JM. Pain management during retinopathy of prematurity eye examinations: a systematic review. Adv Neonatal Care 2009; 9: 99-110.

7 Anand KJS. International Evidence-based Group for Neonatal Pain. Consensus statement for the prevention and management of pain in the newborn. Arch Pediatr Adolesc Med 2001; 155: 173-180.
8 Rush R, Rush S, Nicolau J, Chapman K, Naqvi M. Systemic manifestations in response to mydriasis and physical examination during screening for retinopathy of prematurity. Retina 2004; 24: 242-245.

9 Laws DE, Morton C, Weindling M, Clark D. Systemic effects of screening for retinopathy of prematurity. Br J Ophthalmol 1996; 80: 425-428.

10 Marsh VA, Young WO, Dunaway KK, Kissling GE, Carlos $\mathrm{RQ}$, Jones SM et al. Efficacy of topical anesthetics to reduce pain in premature infants during eye examinations for retinopathy of prematurity. Ann Pharmacother 2005; 39: 829-833.

11 Johnston CC, Stevens B, Pinelli J, Gibbins S, Filion F, Jack A et al. Kangaroo care is effective in diminishing pain response in preterm neonates. Arch Pediatr Adolesc Med 2003; 157: 1084-1088.

12 Chermont AG, Falcao LF, de Souza Silva EH, de Cássia Xavier Balda R, Guinsburg R. Skin-to-skin contact and/or oral $25 \%$ dextrose for procedural pain relief for term newborn infants. Pediatrics 2009; 124: e1101-e1107.

13 Johnston CC, Stevens BJ, Yang F, Horton L. Differential response to pain by very premature neonates. Pain 1995; 61: 471-479.

14 Johnston CC, Stevens BJ, Franck LS, Jack A, Stremler R, Platt R. Factors explaining lack of response to heel stick in preterm newborns. J Obstet Gynecol Neonatal Nurs 1999; 28: 587-594.

15 Craig KD, Whitfield MF, Grunau RVE, Linton J Hadjistavropoulos HD. Pain in the pre-term neonate: behavioral and physiological indices. Pain 1993; 52: 287-299.

16 Porter FL, Wolf CM, Miller JP. Procedural pain in newborn infants: the influence of intensity and development. Pediatrics 1999; 104: e13.

17 Stevens B, Johnston C, Petryshen P, Taddio A. Premature infant pain profile: Development and initial validation. Clinical Journal of Pain 1996; 12: 13-22. 